\title{
A New Arrangement of Active Coils for Wireless Charging of UAV
}

\author{
Zhengwang $\mathrm{He}^{1}{ }^{1}$, Zhiyong $\mathrm{Li}^{1, *}$, Ruoyue Wang ${ }^{1}$, Ying Fan ${ }^{2}$ and Minqian $\mathrm{Xu}^{1}$ \\ 1 School of Automation, Central South University, Changsha 410083, China; 8207181024@csu.edu.cn (Z.H.); \\ 0908170424@csu.edu.cn (R.W.); 1406160104@csu.edu.cn (M.X.) \\ 2 School of Electric Engineering, Guangdong Mechanical \& Electronical College of Technology, \\ Guangzhou 510515, China; 2014010008@gdmec.edu.cn \\ * Correspondence: lizy@mail.csu.edu.cn; Tel.: +86-138-7312-7689
}

Citation: He, Z.; Li, Z.; Wang, R.; Fan, Y.; Xu, M. A New Arrangement of Active Coils for Wireless Charging of UAV. Energies 2021, 14, 5754. https://doi.org/10.3390/en14185754

Academic Editor: José Gabriel Oliveira Pinto

Received: 21 July 2021

Accepted: 9 September 2021

Published: 13 September 2021

Publisher's Note: MDPI stays neutral with regard to jurisdictional claims in published maps and institutional affiliations.

Copyright: (c) 2021 by the authors. Licensee MDPI, Basel, Switzerland. This article is an open access article distributed under the terms and conditions of the Creative Commons Attribution (CC BY) license (https:// creativecommons.org/licenses/by/ $4.0 /)$.

\begin{abstract}
This paper presents the design and optimization of a wireless power transfer (WPT) charging system based on magnetically coupled resonant technology, applied to an Unmanned Aerial Vehicle (UAV). In this paper, a charging system, including dual active transmitter coils and a single receiver coil, is proposed. The dual transmitting coils adopt a coaxial structure with different radii. This structure simplifies the calculation of the complex mutual inductance between the coils to a function of mutual inductance only related to the value of the radial misalignment. Aiming toward a constant charging power, the optimal transmission efficiency of electric energy is achieved by controlling the input voltages of the active coils, which are solved via a set of equations defined as Lagrange multipliers. The simulation results of the $570 \mathrm{~V}$ and $85,000 \mathrm{~Hz}$ system verified the validity of the proposed wireless UAV charging scheme.
\end{abstract}

Keywords: Lagrange multiplier; magnetically coupled resonant technology; power compensation; radial misalignment

\section{Introduction}

UAVs undoubtedly have high research potential and application value, but the flight time of UAVs restricts their development. This is because a high-energy density lithium battery, which is adopted to the UAV, only permits a flight time of about 20-40 min [1].

Thus, researchers began to research the application of magnetically coupled resonant technology to charge UAVs for solving this limitation [2-5]. This method creates a base station where the batteries can be automatically charged after landing [3]. The proposed magnetically coupled resonant system in [2] is mainly composed of two coils, namely, a single transmitting coil and a single receiving coil. The power transmission efficiency between the two coils is a function of the vertical distance between the two coils and the power supply. When the two coils are coaxial, the power transmission efficiency of the coil is the highest, and when the distance between the two coils is increased, the power transmission efficiency decreases. In the practical charging process of UAVs, the coaxial position of the receiving coil and the transmitting coil cannot be guaranteed, namely, radial misalignment, which will lead to a reduction in output power and charging time delay [6]. Therefore, it is necessary to research the power compensation problem of misaligned charging.

Scholars have adopted various methods to solve this problem [7-18]. In [7-11], a reconfigurable resonator and numerous transmission coil arrays with different structures have been used to improve transmission efficiency. A relatively complete mutual inductance model for analyzing magnetically coupled resonant technology was established. A planar transmitting resonator with an array of $2 \times 2$ using a single feeding loop was presented in [12]. This structure could maintain high transfer efficiency in a plane. However, the power transfer efficiency function determined by this structure is still two-dimensional 
in essence. This leads to the need for complex control strategies to achieve the goal of constant output power, which is not worth it in practical application. In [13], two circular planar spiral coils were applied to both receive and transmit circuits of a WPT system. The main disadvantage of this configuration is that it reduces the load capacity of the UAV. In [14], an automatic landing procedure for UAV was proposed, which fundamentally reduces the possibility of radial misalignment. A WPT charging system with a movable transmitting coil was introduced in [15]. When the UAV lands, the mobile transmitting coil can automatically align with the receiving coil. Of course, it requires a high-precision positioning system. A lightweight wireless charging system was presented in [17]. This design can improve the load capacity of UAVs without affecting the operation of various equipment carried by UAVs. In [18], Noriaki Oodachi et al. have proposed a wireless power transmission system to solve this problem by using phase weights of transmission coil arrays. When the receiving coil is not coaxial with the transmitting coil, according to the direction between the transmission coil and the receiving coil, the transmission coil of the coil array is excited by the transmission circuit and the corresponding phase weight to achieve the purpose of power compensation. However, this method also requires a large number of coils, and the control algorithm and implementation are complex, which increase the costs.

The system proposed in this paper is simple and effective. In order to compensate the power reduction caused by radial misalignment, two coaxial active coils with different radii are used as the transmitting coils, and the compensation is realized by controlling the different input power of the two coils. When the output power of one transmitting coil decreases due to radial misalignment, the other transmitting coil with different radii that are still coaxial is applied to compensate the output power, so as to maximize the transmission efficiency of the system, which satisfies the Lagrange multiplier. In addition, by controlling the input voltage of the two active coils of the system, a power supply with arbitrary output power can be designed. This design simplifies the efficiency function from a function with two-dimensional variables to a function with only a radial variable, so there is no need for a complex control algorithm, which makes it more practical.

\section{Proposed Wireless Charging of UAV System}

This paper presents the design of a UAV charging system with magnetically coupled resonant technology, as shown in Figure 1. The transmitting coil uses double coaxial active coils, with the receiving coil and transmitting coils coupled by air. The vertical distance between the UAV and the charging pile is $10 \mathrm{~cm}$. Both the double transmitting circuit and the single receiving circuit adopt the topology of inductance and capacitor in series. The whole system realizes power transmission through the full resonance of coil and capacitor.

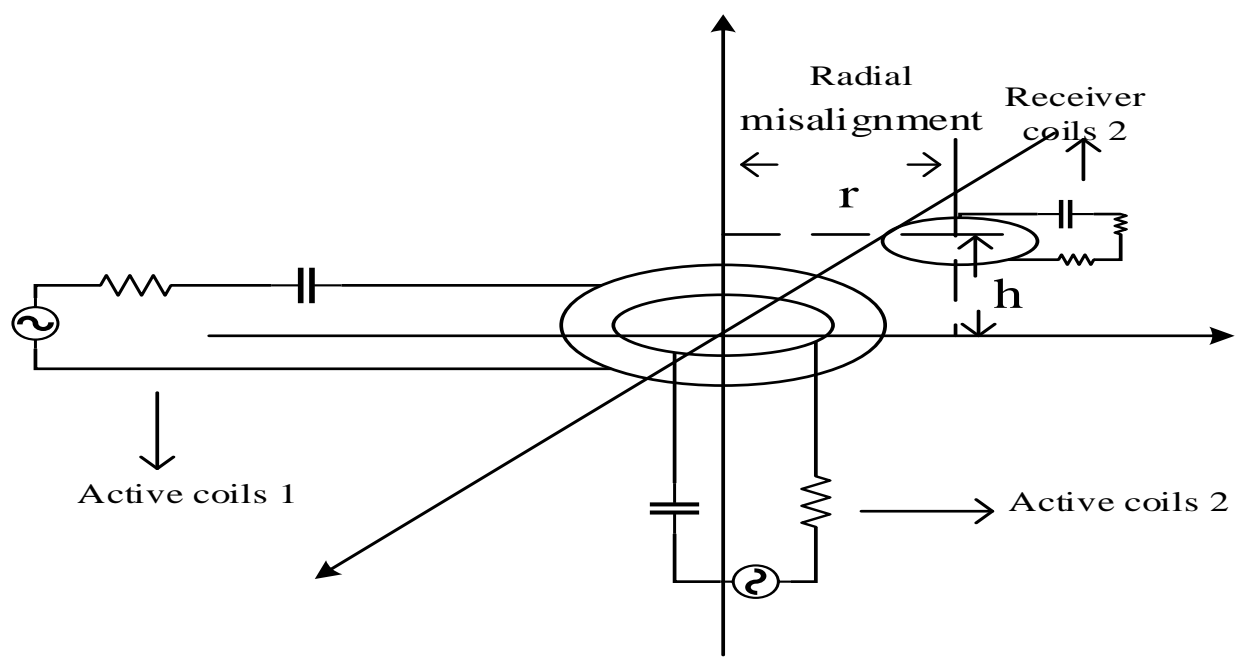

Figure 1. Proposed system for UAV charging. 
In order to research the specific internal strategy of the new charging pile, two mathematical models, including the power model and mutual inductance model of the system, are established according to the actual charging situation of the UAV, and the theoretical analysis is carried out. The power model analyzes the relationship between the output power of the system and the input voltage of the two active coils; the transmission efficiency is found when the mutual inductance and output power are constant. The mutual inductance model analyzes the relationship between the mutual inductance of the system and the radial misalignment caused by the landing error when the UAV is charging, and provides a scheme to compensate for the system's output power reduction that is simple and effective. A $570 \mathrm{~V}, 85,000 \mathrm{~Hz}$ system is built in MATLAB/Simulink, and the output power of the system is obtained. The transmission efficiency of the system can reach $82 \%$. At the same time, it is confirmed that the double transmitting coils have a good compensation effect on the power reduction caused by the radial misalignment of the system; the correctness of the control strategy is also verified.

\subsection{Model of System Mutual Inductance and Radial Misalignment Distance}

In most of the experimental studies, the transmitting coil and receiving coil are placed in parallel, and their central axis position is the same. However, when the UAV actually stops, it cannot be guaranteed that it will stop in the axial direction of the transmitting coil. When the position of the coil deviates, the mutual inductance $\mathrm{M}$ will change. When the system frequency is fixed and the coil resistance is constant, the change in mutual inductance $\mathrm{M}$ is the most important factor affecting system performance. Therefore, when the receiving coil has radial misalignment, the mutual inductance between the receiving coil and the transmitting coil changes, which leads to a change in system power and efficiency.

Only the mutual inductance mode of the single transmitting and single receiving coils is considered, as shown in Figure 2. The center of the transmitting coil is placed in the coordinate system $(0,0,0)$, and the center of the receiving coil is placed in the coordinate system $(0, t, h)$.

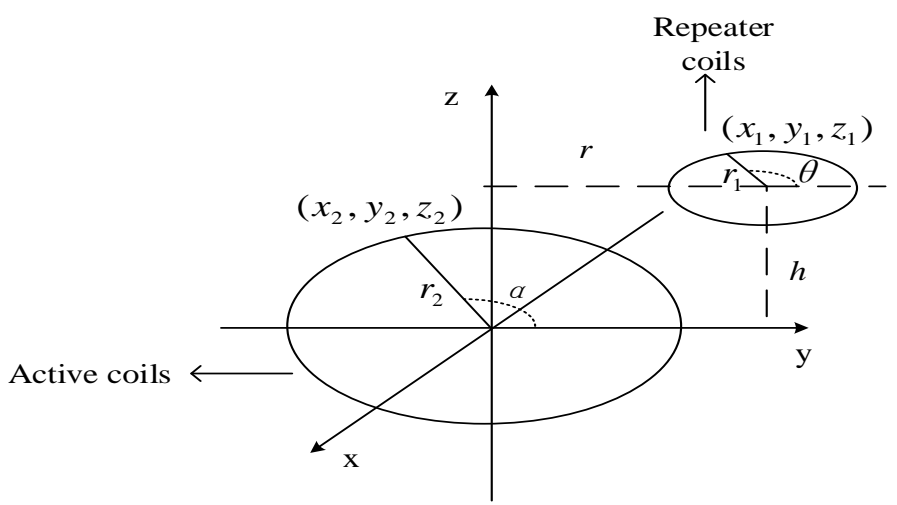

Figure 2. One active to receiver charging mode.

The parameter equations of the transmitting and receiving coils are listed as follows:

$$
\left\{\begin{array}{c}
x_{1}=r_{1} \times \cos \theta \\
y_{1}=r_{1} \times \sin \theta+r \\
z_{1}=h \\
x_{2}=r_{2} \times \cos \alpha \\
y_{2}=r_{2} \times \sin \alpha
\end{array}\right.
$$

Therefore:

$$
\left\{\begin{array}{c}
\mathrm{d} l_{1}=\left(r_{1} \times \cos \theta-r_{1} \times \sin \theta\right) \mathrm{d} \theta \\
\mathrm{d} l_{2}=\left(r_{2} \times \cos \alpha-r_{2} \times \sin \alpha\right) \mathrm{d} \alpha \\
\mathrm{d} l_{1} \times \mathrm{d} l_{2}=r_{1} \times r_{2} \cos (\theta-\alpha) \mathrm{d} \theta \mathrm{d} \alpha
\end{array}\right.
$$


According to the Neumann formula, when the number of turns of the receiving coil is $N_{1}$ and the number of turns of the transmitting coil is $N_{2}$, the mutual inductance formula between them is as follows:

$$
M=\frac{N_{1} \times N_{2}}{4 \pi} \times \frac{\mathrm{d} l_{1} \times \mathrm{d} l_{2}}{R}=\frac{N_{1} \times N_{2}}{4 \pi} \int_{0}^{2 \pi} \int_{0}^{2 \pi} \frac{r_{1} \times r_{2} \cos (\theta-\alpha) \mathrm{d} \theta \mathrm{d} \alpha}{D}
$$

In the above formula:

$$
\mathrm{D}=\sqrt{\left(r_{1} \times \cos \theta-r_{2} \times \cos \alpha\right)^{2}+\left(r_{1} \times \sin \theta+r-r_{2} \times \sin \alpha\right)^{2}+h^{2}}
$$

Analysis of Equation (3) shows that the mutual inductance of the system decreases with the increase in radial misalignment, and the output power decreases with the decrease in mutual inductance. Therefore, it is necessary to compensate for the mutual inductance reduction caused by the radial misalignment of the single transmitter and single receiver system.

Considering double transmitting and single receiving coils, the two transmitting coils are coaxial with different radii. With the increase in radial misalignment, the mutual inductance between the two transmitting coils and the receiving coil changes according to Equation (3). However, for the receiving coil, the total mutual inductance is the sum of mutual inductance between the two transmitting coils and the receiving coil, which compensates for the reduction in mutual inductance; Maxwell simulation verifies this conclusion.

\subsection{Model of Output Power and Input Voltage}

We define the mutual inductance between coil 1 and coil 2 as $M_{12}$, and define $M_{13}$ and $M_{23}$ in the same way. When the UAV lands on the charging pile, the relative position between the receiving coil and the two transmitting coils is fixed, so the mutual inductance between the two coils is fixed, i.e., $M_{13}, M_{23}$ and $M_{12}$ are constant. The output power of the system is only related to the input voltage of the active coil, as shown in Figure 3. The detailed model analysis is as follows:

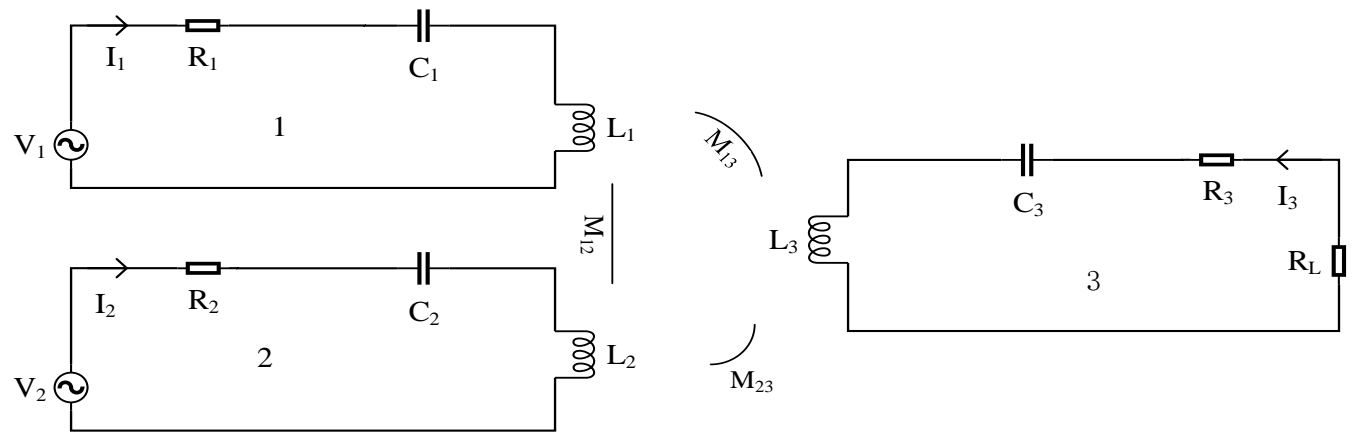

Figure 3. Two active to receiver charging modes.

In order to simplify the model, the parameters of the circuit model are symmetrical, namely, $R_{1}=R_{2}=R_{3}=R$. The transmitter and receiver of the system will work at the same frequency, which is defined as:

$$
\omega=2 \pi \mathrm{f}=\frac{1}{\sqrt{\mathrm{C}_{1} \mathrm{~L}_{1}}}=\frac{1}{\sqrt{\mathrm{C}_{2} \mathrm{~L}_{2}}}=\frac{1}{\sqrt{\mathrm{C}_{3} \mathrm{~L}_{3}}}
$$

where $\mathrm{f}$ is the fundamental frequency of the power supply.

By listing the voltage equation of each circuit, the following voltage and current (Equation (5)) can be obtained: 


$$
\left(\begin{array}{c}
V_{1} \\
V_{2} \\
0
\end{array}\right)=\left(\begin{array}{ccc}
\mathrm{R}_{1}+j \omega \mathrm{L}_{1}+\frac{1}{j \omega \mathrm{C}_{1}} & -j \omega M_{12} & -j \omega M_{13} \\
-j \omega M_{12} & \mathrm{R}_{2}+j \omega \mathrm{L}_{2}+\frac{1}{j \omega C_{2}} & -j \omega M_{23} \\
-j \omega M_{13} & -j \omega M_{23} & R_{3}+R_{1}+j \omega L_{3}+\frac{1}{j \omega C_{3}}
\end{array}\right)\left(\begin{array}{c}
I_{1} \\
I_{2} \\
I 3
\end{array}\right)
$$

The input impedance of the system is solved. According to the defined system operating frequency, order $V_{2}=0$, the following equation (Equation (6)) can be obtained:

$$
Z_{\text {sent } 1}=R_{1}+\frac{\left(\omega M_{12}\right)^{2}\left(R_{3}+R_{1}\right)-j \omega^{3} M_{12} M_{13} M_{23}}{R_{2}\left(R_{3}+R_{1}\right)+\left(\omega M_{23}\right)^{2}}+\frac{\left(\omega M_{13}\right)^{2} R_{2}+j \omega^{3} M_{12} M_{13} M_{23}}{R_{2}\left(R_{3}+R_{1}\right)+\left(\omega M_{23}\right)^{2}}=R_{1}+Y_{2}+Y_{3}
$$

$Y_{2}$ and $Y_{3}$ in Equation (6), respectively, indicate the mutual inductance influence of loop 2 and loop 3 on loop 1 :

$$
\left\{\begin{array}{c}
Y_{2}=\frac{\left(\omega M_{12}\right)^{2}\left(R_{3}+R_{1}\right)-j \omega^{3} M_{12} M_{13} M_{23}}{R_{2}\left(R_{3}+R_{1}\right)+\left(\omega M_{23}\right)^{2}} \\
Y_{3}=\frac{\left(\omega M_{13}\right)^{2} R_{2}+j \omega^{3} M_{12} M_{13} M_{23}}{R_{2}\left(R_{3}+R_{1}\right)+\left(\omega M_{23}\right)^{2}}
\end{array}\right.
$$

Therefore, the input current $I_{1}$ of active coil 1 can be expressed as:

$$
I_{1}=\frac{V_{1}}{Z_{\text {sent } 1}}=\frac{V_{1}}{R_{1}+Y_{2}+Y_{3}}
$$

Aligned, the input current $I_{2}$ of active coil 2 can be expressed as:

$$
I_{2}=\frac{V_{2}}{Z_{\text {sent } 2}}=\frac{V_{2}}{R_{2}+Y_{2}^{\prime}+Y_{3}^{\prime}}
$$

Among:

$$
\left\{\begin{array}{c}
Y_{2}^{\prime}=\frac{\left(\omega M_{12}\right)^{2}\left(R_{3}+R_{1}\right)-j \omega^{3} M_{12} M_{13} M_{23}}{R_{1}\left(R_{3}+R_{1}\right)+\left(\omega M_{13}\right)^{2}} \\
Y_{3}^{\prime}=\frac{\left(\omega M_{23}\right)^{2} R_{1}+j \omega^{3} M_{12} M_{13} M_{23}}{R_{1}\left(R_{3}+R_{1}\right)+\left(\omega M_{13}\right)^{2}}
\end{array}\right.
$$

Considering two active coils, the output current $I_{3}$ of the receiver can be expressed as:

$$
I_{3}=\frac{j \omega M_{13} \times I_{1}+j \omega M_{23} \times I_{2}}{R_{3}+R_{1}}=\frac{j \omega M_{13}}{R_{3}+R_{1}} \times \frac{V_{1}}{R_{1}+Y_{2}+Y_{3}}+\frac{j \omega M_{23}}{R_{3}+R_{1}} \times \frac{V_{2}}{R_{2}+Y_{2}^{\prime}+Y_{3}^{\prime}}
$$

Among $Z_{\text {sent } 2}=R_{1}+Y_{2}{ }^{\prime}+Y_{3}{ }^{\prime}$. Therefore, the input power $P_{\text {in }}$ and output power $P_{\text {out }}$ of the system can be expressed as:

$$
\begin{gathered}
P_{\text {in }}=\operatorname{Re}\left(V_{1} \times I_{1}^{*}\right)+\operatorname{Re}\left(V_{2} \times I_{2}^{*}\right)=\frac{\left(V_{1}\right)^{2}}{\mathrm{R}_{1}+Y_{2}+Y_{3}}+\frac{\left(V_{2}\right)^{2}}{\mathrm{R}_{2}+Y_{2}{ }^{\prime}+Y_{3}{ }^{\prime}} \\
P_{\text {out }}=I_{3}^{2} \times \mathrm{R}_{1}=\left(\frac{\mathrm{j} \omega}{\left(\mathrm{R}_{3}+\mathrm{R}_{1}\right)}\right)^{2} \times\left(\frac{M_{13} V_{1}}{\mathrm{R}_{1}+Y_{2}+Y_{3}}+\frac{M_{23} V_{2}}{\mathrm{R}_{2}+Y_{2}{ }^{\prime}+Y_{3}{ }^{\prime}}\right)^{2} \times \mathrm{R}_{1}
\end{gathered}
$$

Therefore, the efficiency of the system $\eta$ can be calculated as follows:

$$
\eta=\frac{P_{\text {out }}}{P_{\text {in }}}=\left(\frac{j \omega}{R_{3}+R_{1}}\right)^{2} \times \frac{Z_{\text {sent } 1} \times Z_{\text {sent } 2} \times\left(\frac{M_{13} V_{1}}{Z_{\text {sent1 }}}+\frac{M_{23} V_{2}}{Z_{\text {sent2 }}}\right)^{2}}{\left(V_{1}\right)^{2} \times Z_{\text {sent } 2}+\left(V_{2}\right)^{2} \times Z_{\text {sent1 }}} \times R_{1}
$$

Thus, the value of $\eta$ is related to the two input voltages of the system and the mutual inductance between the two coils. Additionally, the observation Equations (13) and (14) show that when the output power and radial misalignment are fixed, there must be the maximum transmission efficiency. 
The output power $P_{\text {out }}$ is set as constant at $640 \mathrm{~W}$. The goal is to maximize transmission efficiency. The optimal problem formulation is Equation (15):

$$
\left\{\begin{array}{c}
\operatorname{Max}:\left(\eta \eta=\frac{P_{\text {out }}}{P_{\text {in }}}=\left(\frac{j \omega}{R_{3}+R_{1}}\right)^{2} \times \frac{Z_{\text {sent } 1} \times Z_{\text {sent } 2} \times\left(\frac{M_{13} V_{1}}{Z_{\text {sent } 1}}+\frac{M_{23} V_{2}}{Z_{\text {sent } 2}}\right)^{2}}{\left(V_{1}\right)^{2} \times Z_{\text {sent } 2}+\left(V_{2}\right)^{2} \times Z_{\text {sent } 1}} \times R_{1}\right) \\
P_{\text {out }}-640=0
\end{array}\right\}
$$

Lagrange multiplier $\lambda$ is introduced and a Lagrangian function is constructed:

$$
L=\eta-\lambda \times\left(P_{\text {out }}-640\right)
$$

When the partial derivative of Equation (16) is found, they can be set to zero:

$$
\left\{\begin{array}{c}
\frac{\partial L}{\partial V_{1}}=\frac{\partial \eta}{\partial V_{1}}-\lambda \times \frac{\partial P_{\text {out }}}{\partial V_{1}}=0 \\
\frac{\partial L}{\partial V_{2}}=\frac{\partial \eta}{\partial V_{2}}-\lambda \times \frac{\partial P_{\text {out }}}{\partial V_{2}}=0 \\
P_{\text {out }}=640
\end{array}\right.
$$

The corresponding $V_{1}$ and $V_{2}$ are obtained when the efficiency is maximum, as long as $V_{1}$ and $V_{2}$ are satisfied (Equation (17)).

The flowchart of the methodology mentioned is introduced in Figure 4.

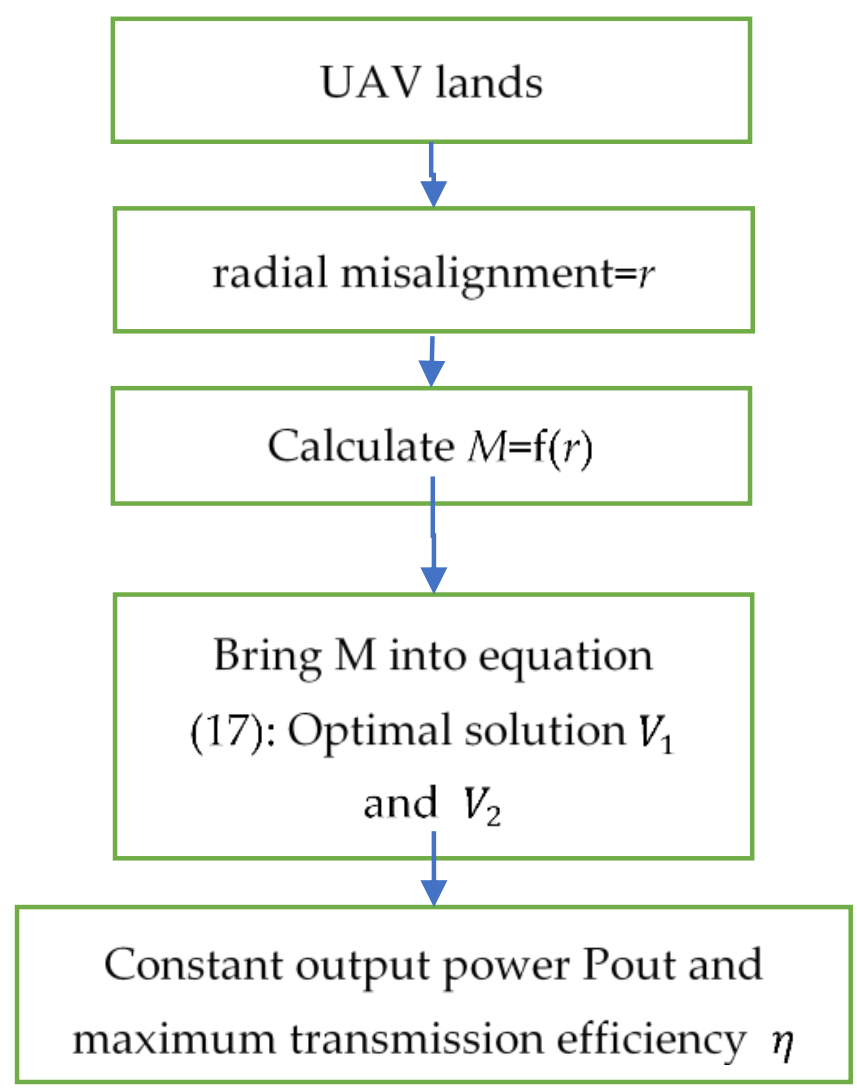

Figure 4. The flowchart of the methodology.

\section{Simulation}

The proposed $570 \mathrm{~V}$ and 85,000 Hz power model is simulated in MATLAB/Simulink, and the rationality of the model is verified. The parameters of the system are listed in Table 1. The mutual inductance parameters of the coil are simulated by the finite element method in Maxwell. 
Table 1. Parameters of the system.

\begin{tabular}{cccc}
\hline Parameter & Value & Parameter & Value \\
\hline $\mathrm{L}_{1} \& \mathrm{~L}_{3}$ & $500.0 \mu \mathrm{H}$ & Peak amplitude of $V_{1} \& V_{2}$ & $570 \mathrm{~V}$ \\
$\mathrm{C}_{1} \& \mathrm{C}_{3}$ & $0.0072 \mu \mathrm{F}$ & $\mathrm{f}$ & $85,000 \mathrm{~Hz}$ \\
$\mathrm{R}_{1} \& \mathrm{R}_{2} \& \mathrm{R}_{3}$ & $0.2 \Omega$ & $\mathrm{M}_{13}$ & $175 \mu \mathrm{H}$ \\
$\mathrm{R}_{\mathrm{L}}$ & $20 \Omega$ & $M_{23}$ & $300 \mu \mathrm{H}$ \\
$M_{12}$ & $400 \mu \mathrm{H}$ & $\mathrm{C}_{2}$ & $0.0036 \mu \mathrm{F}$ \\
$\mathrm{L}_{2}$ & $1000 \mu \mathrm{H}$ & & \\
\hline
\end{tabular}

\subsection{Simulation of Mutual Inductance Parameters}

When the coil has radial misalignment, double transmitting and single receiving coils are considered. The radii of the two transmitting coils are $26 \mathrm{~cm}$ and $36 \mathrm{~cm}$, respectively, and the radius of the receiving coil is $26 \mathrm{~cm}$. The vertical distance between the transmitting coil and the receiving coil is kept at $10 \mathrm{~cm}$, and the medium is air. The finite element simulation, as shown in Figure 5a, is carried out in Maxwell. The relationship between mutual inductance and radial distance in the two transmitting coils and receiving coils can be obtained, as shown in Figure 5b,c. It is found that the mutual inductance between the two transmitting coils and the radial distance decreases with the increase in radial misalignment. Combined with the power model, the power of the system also decreased. For the receiving coil, mutual inductance is the sum of the two, which compensates for the reduction in mutual inductance and power of the single transmitting coil. At the same time, the self-inductance of the $36 \mathrm{~cm}$ coil is twice that of the other two $26 \mathrm{~cm}$ coils, and the self-inductance is only related to the material and radius of the coil.

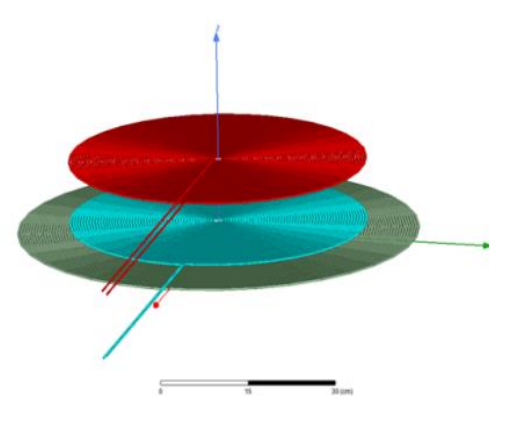

(a) Maxwell simulation diagram inductance and radial distance of $26 \mathrm{~cm}$ transmitting coil

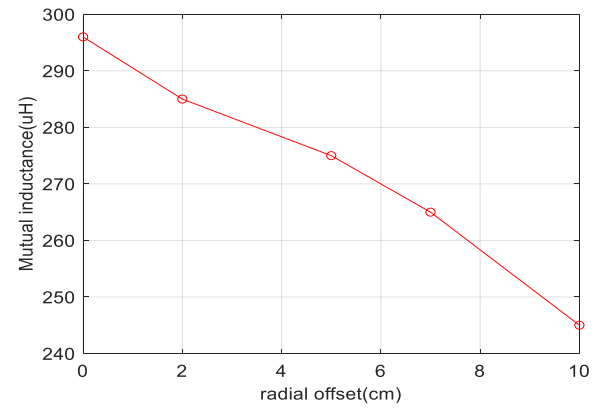

(c) Relationship between mutual inductance and radial distance of $36 \mathrm{~cm}$ transmitting coil

Figure 5. Maxwell simulation. (a) Snapshot of Maxwell simulation diagram (b) Simulation result of $26 \mathrm{~cm}$ transmitting coil. (c) Simulation result of $36 \mathrm{~cm}$ transmitting coi.l.

\subsection{Power}

According to the coil parameters calculated in model A, the simulation output waveform of the system simulated by Simulink is shown in Figure 6. A snapshot of the simulation file is shown in Figure 6a. We use a transformer to replace the resonant coils and their mutual inductance. As for the high-frequency AC power required by the system, we directly use AC power to generate it. Both the double transmitting circuit and single receiving circuit adopt the topology of inductance and capacitor in series. In other figures, $U_{\text {out }}$ is the output voltage waveform. $U_{\text {in }}$ is the input voltage of the active coil. $I_{\text {in }}$ is the input current waveform of the active coil. $I_{o u t}$ is the output current waveform. Output power is the output active power of the system and input power1 and input power2 are the input active power of the system. According to the simulated voltage and current, the transmission efficiency of the system can be calculated, which reaches $94 \%$. Comparing the simulation results with the theoretical results, the output power error is less than $5 \%$. 
At the same time, the simulation results show that the output current and voltage waveform is sine wave, but there are phase differences, which is caused by mutual inductance resonance not being complete, and therefore, there is phase lag.

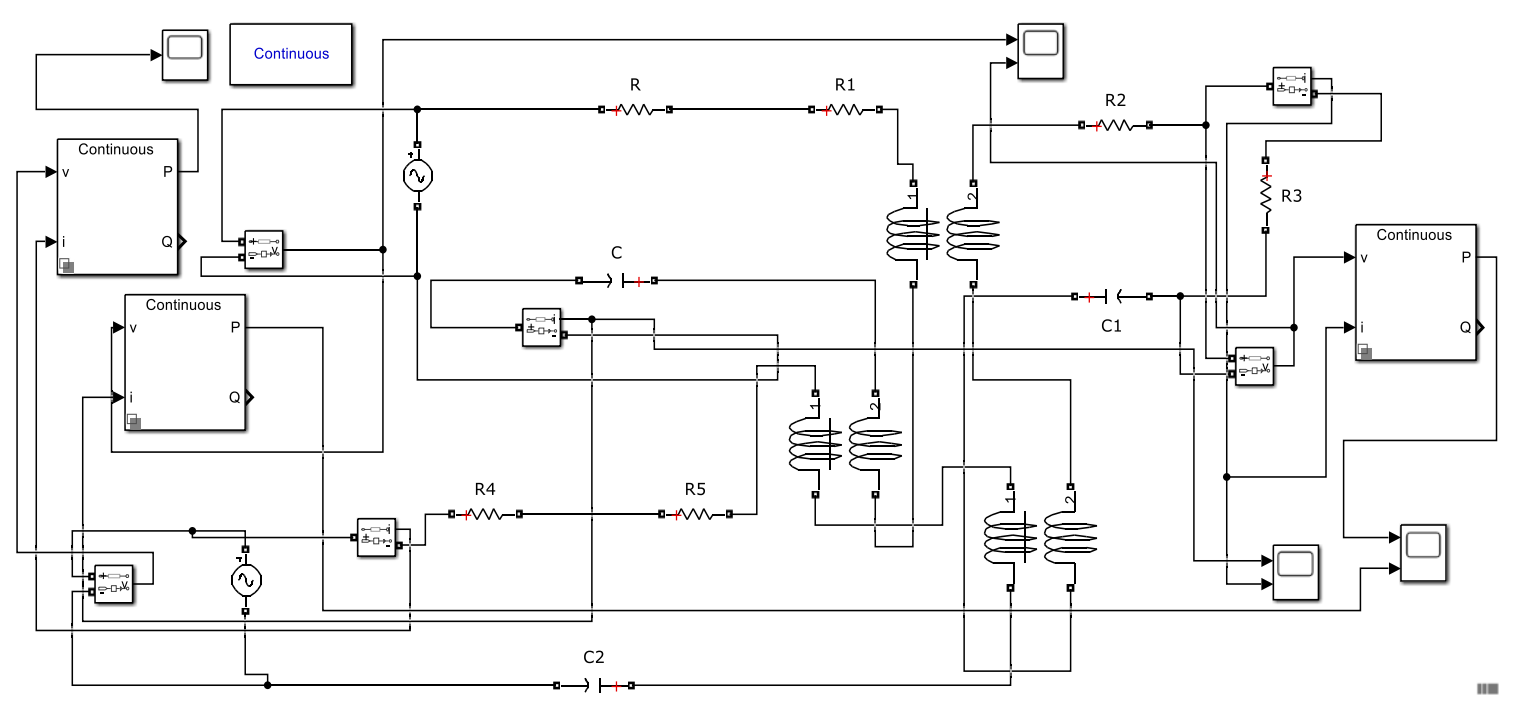

(a) One snapshot of the simulation file
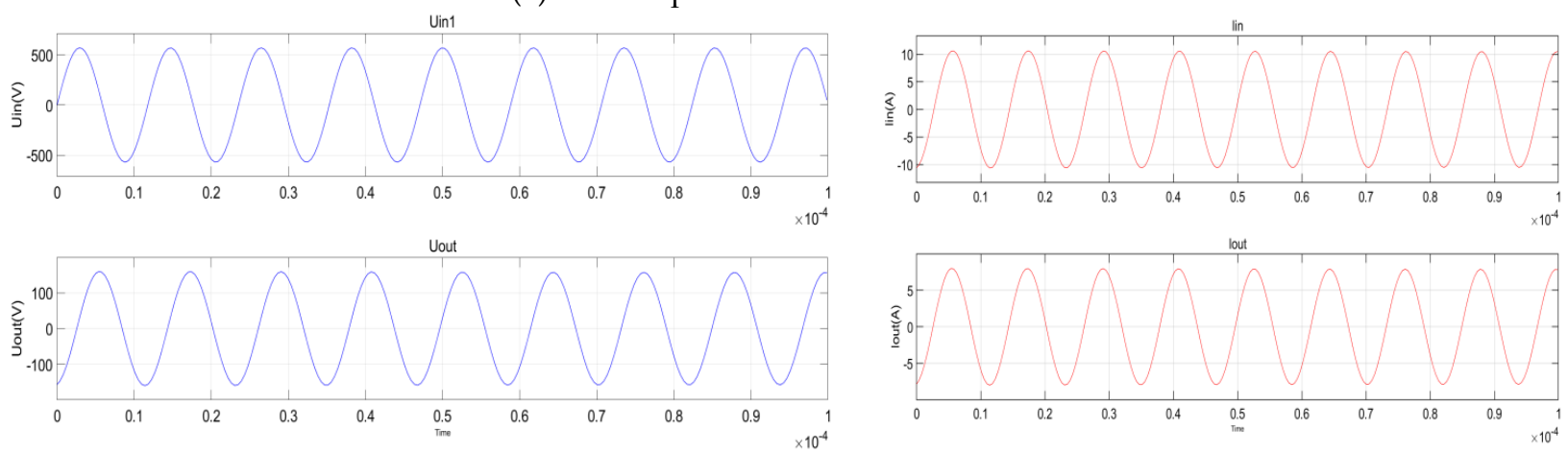

(b) Two to one system: the input voltage and the load voltage

(c) The input current and the load current

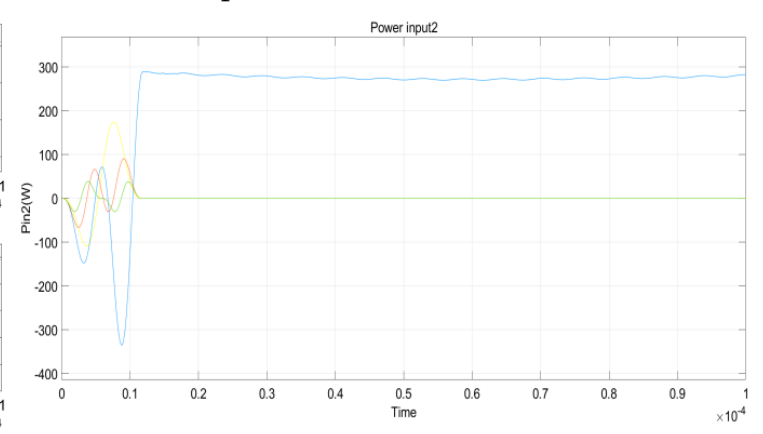

(e) The input power2

(d) The output power and the input power1

Figure 6. Waveforms of the transmitter currents and the load voltage. (a) Snapshot of Matlab simulation (b) Voltage (c) Current (d) Output power and input power 1 (e) input power 2.

By changing the value of the input voltage, an arbitrary power output can be achieved. Table 2 shows the simulated output power under different voltage peaks, which is close to the theoretically derived value.

In addition, different radial misalignment will cause a decrease in the mutual inductance between the receiving coil and the transmitting coil, resulting in a decrease in power. As shown in Figure 7, when the peak input voltages of the two active coils are both $570 \mathrm{~V}$, the output power is negatively correlated with the radial misalignment. 
Finally, when the radial misalignment is constant with the input voltage of one active coil, the simulation between the output power and the input voltage of the other active coil verifies the correctness of the control strategy.

The verification result is shown in Figure 8. At this time, the radial misalignment of the system is $5 \mathrm{~cm}$, and the input voltage of an active coil is $570 \mathrm{~V}$. The output power of the system increases as the input voltage of the active coil increases.

Table 2. Comparison of simulation and theoretical values of output power.

\begin{tabular}{|c|c|c|c|c|c|}
\hline & Value & Value & Value & Value & Value \\
\hline $\begin{array}{l}\text { Peak Amplitude of Input Voltage } \\
\qquad\left(V_{1}=V_{2}\right)(\mathrm{V})\end{array}$ & 300 & 400 & 500 & 570 & 600 \\
\hline $\begin{array}{c}\text { Simulation } \\
(\mathrm{W})\end{array}$ & 180 & 310 & 500 & 640 & 710 \\
\hline $\begin{array}{l}\text { Theoretical } \\
\text { (W) }\end{array}$ & 186 & 320 & 525 & 662 & 739 \\
\hline
\end{tabular}

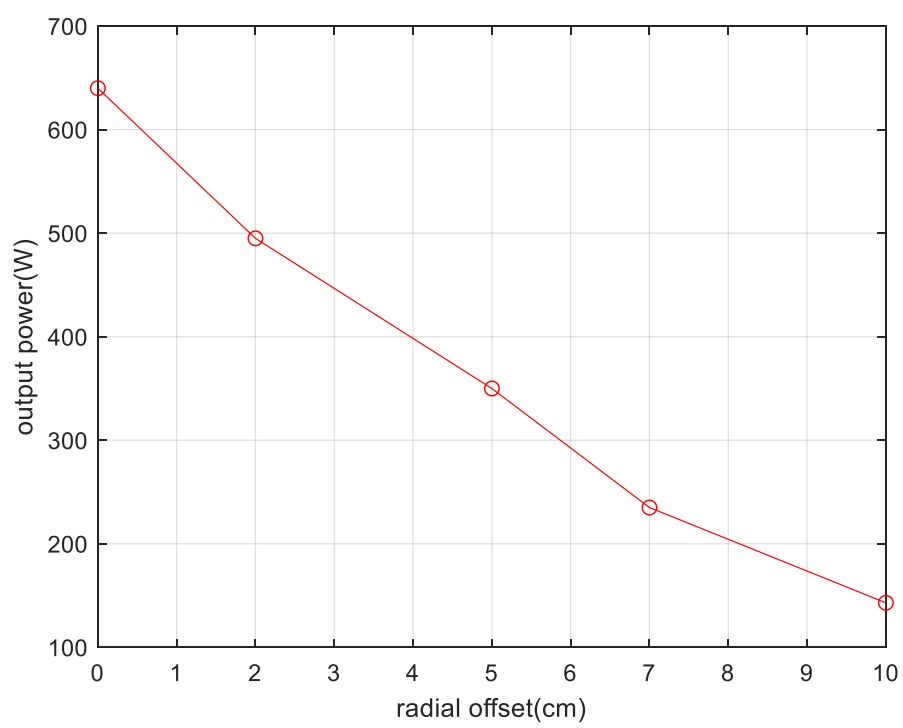

Figure 7. The output power and the radial misalignment.

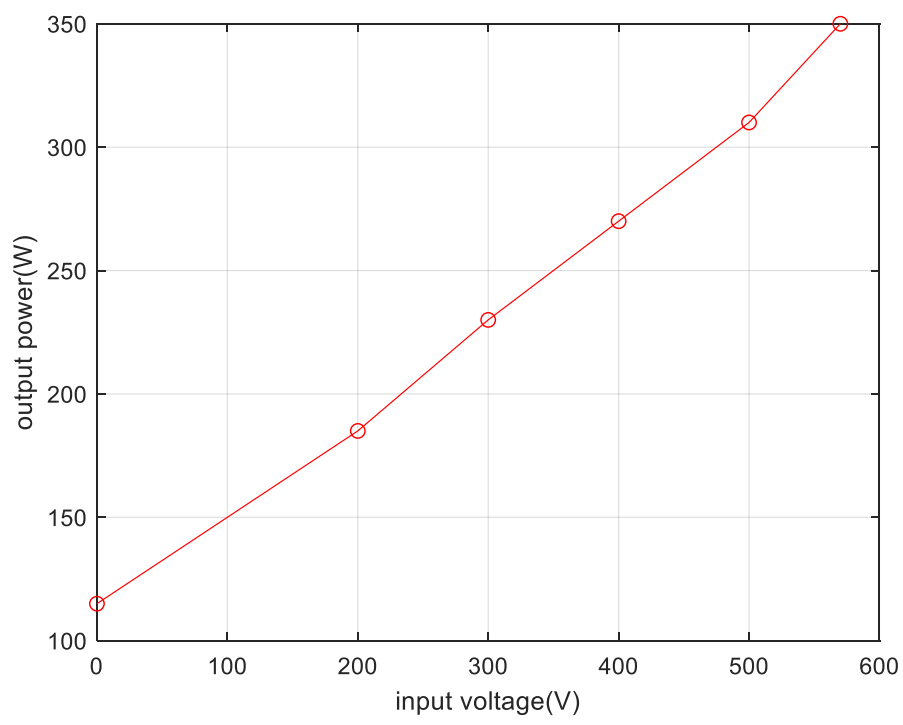

Figure 8. The output power and the input voltage. 


\section{Discussion}

This paper presents a new wireless power transmission charging system for UAVs, and establishes two mathematical models to perfect the theoretical basis of the system. We also determine the control strategy between output power and input voltage when there is radial misalignment. The structure of a double transmitting coil and single receiving coil can better compensate for the mutual inductance and output power drop caused by radial misalignment. At the same time, the output power of the system can be changed by controlling the input voltage of the system to realize a power supply of any output power, which is simple and easy to operate. When the output power is constant, maximum power transfer efficiency is obtained by controlling the input voltage of the two coils. Numerical and simulation results have been presented in terms of electrical performance to demonstrate the validity of the proposed structure.

Author Contributions: Conceptualization, Z.H. and Z.L.; methodology, Z.H.; software, Z.H.; validation, M.X., Y.F. and R.W.; formal analysis, Z.H.; investigation, M.X.; resources, Z.L.; data curation, R.W.; writing—original draft preparation, Z.H.; writing—review and editing, Z.H.; visualization, R.W.; supervision, Z.L.; project administration, Z.H.; funding acquisition, Z.L. All authors have read and agreed to the published version of the manuscript.

Funding: This research received no external funding.

Data Availability Statement: The data presented in this study are available on request from the corresponding author.

Acknowledgments: Thanks are due to Wenjing Xiong and Zixi Liu, of Central South University, for their help in the testing.

Conflicts of Interest: The authors declare no conflict of interest.

\section{References}

1. Lee, B.; Kwon, S.; Park, P.; Kim, K. Active power management system for an unmanned aerial vehicle powered by solar cells, a fuel cell, and batteries. IEEE Trans. Aerosp. Electron. Syst. 2014, 50, 2253-2268. [CrossRef]

2. Kurs, A.; Karalis, A.; Moffatt, R.; Joannopoulos, J.D.; Fisher, P.; Soljačic, M. Wireless Power Transfer via Strongly Coupled Magnetic Resonances. Science 2007, 317, 83-86. [CrossRef] [PubMed]

3. Lee, D.; Zhou, J.; Lin, W.T. Autonomous Battery Swapping System for Quadcopter. In Proceedings of the 2015 International Conference on Unmanned Aircraft Systems (ICUAS), Denver, CO, USA, 9-12 June 2015; pp. 118-124.

4. Tak, Y.; Park, J.; Nam, S. Mode-Based Analysis of Resonant Characteristics for Near-Field Coupled Small Antennas. IEEE Antennas Wirel. Propag. Lett. 2009, 8, 1238-1241.

5. Ishizaki, T.; Komori, T.; Ishida, T.; Awai, I. Comparative study of coil resonators for wireless power transfer system in terms of transfer loss. IEICE Electron. Express 2010, 7, 785-790. [CrossRef]

6. Lee, S.G.; Hoang, H.; Choi, Y.H.; Bien, F. Efficiency improvement for magnetic resonance based wireless power transfer with axial-misalignment. Electron. Lett. 2012, 48, 339-340. [CrossRef]

7. Lee, H.H.; Kang, S.H.; Jung, C.W. MR-WPT with reconfigurable resonator and ground for laptop application. IEEE Microw. Wirel. Compon. Lett. 2018, 28, 269-271. [CrossRef]

8. Zhengmin, Z.; Zhiyong, L. Research on the Double Load Performance of Magnetic Coupled Resonant Wireless Power Transfer; Central South University: Changsha, China, 2020.

9. Alshhawy, S.; Barakat, A.; Yoshitomi, K.; Pokharel, R.K. Separation-Misalignment Insensitive WPT System Using Two-Plane Printed Inductors. IEEE Microw. Wirel. Compon. Lett. 2019, 29, 683-686. [CrossRef]

10. Jeon, S.-J.; Seo, D.W. Maximum Output Power Improvement Using Negative Coil in Over-Coupled WPT System. IEEE Microw. Wirel. Compon. Lett. 2020, 30, 810-813. [CrossRef]

11. Kang, S.H.; Choi, J.H.; Harackiewicz, F.J.; Jung, C.W. Magnetic Resonant Three-Coil WPT System Between Off/In-Body for Remote Energy Harvest. IEEE Microw. Wirel. Compon. Lett. 2016, 26, 741-743. [CrossRef]

12. Jolani, F.; Yu, Y.; Chen, Z.D. Electromagnetic Modeling and Optimization of Magnetic Resonant Coupling Wireless Power Transfer Using Coil Array. In Proceedings of the IEEE MTT-S International Conference on Numerical Electromagnetic and Multiphysics Modeling and Optimization (NEMO), Ottawa, ON, Canada, 11-14 August 2015.

13. Campi, T.; Dionisi, F.; Cruciani, S.; De Santis, V.; Feliziani, M.; Maradei, F. Magnetic Field Levels in Drones Equipped with Wireless Power Transfer Technology. In Proceedings of the 2016 Asia-Pacific International Symposium on Electromagnetic Compatibility (APEMC), Shenzhen, China, 17-21 May 2016; pp. 544-547.

14. Junaid, A.B.; Konoiko, A.; Zweiri, Y.; Sahinkaya, M.N.; Seneviratne, L. Autonomous Wireless Self-Charging for Multi-Rotor Unmanned Aerial V ehicles. Energies 2017, 10, 803. [CrossRef] 
15. Choi, C.H.; Jang, H.J.; Lim, S.G.; Lim, H.C.; Cho, S.H.; Gaponov, I. Automatic Wireless Drone Charging Station Creating Essential Environment for Continuous Drone Operation. In Proceedings of the 2016 International Conference on Control, Automation and Information Sciences (ICCAIS), Ansan, Korea, 26-29 October 2016; pp. 132-136.

16. He, X.; Bito, J.; Tentzeris, M.M. A Drone-Based Wireless Power Transfer and Communications Platform. In Proceedings of the 2017 IEEE Wireless Power T ransfer Conference (WPTC), Taipei, Taiwan, 10-12 May 2017; pp. 1-4.

17. Campi, T.; Cruciani, S.; Feliziani, M. Wireless Power Transfer Technology Applied to an Autonomous Electric UAV with a Small Secondary Coil. Energies 2018, 11, 352. [CrossRef]

18. Oodachi, N.; Ogawa, K.; Kudo, H.; Shoki, H.; Obayashi, S.; Morooka, T. Efficiency Improvement of Wireless Power Transfer Via Magnetic Resonance Using Transmission Coil Array. In Proceedings of the IEEE International Symposium on Antennas and Propagation (APSURSI), Spokane, WA, USA, 3-8 July 2011; pp. 1707-1710. 